\title{
Ein neuer grossvolumiger Wasserschöpfer für den Fang von Zooplankton
}

\author{
ABSTRACT \\ A new large volume water sampler for catchment of zooplankton \\ A 90 -liter water sampler for catchment of zooplankton is described. The apparatus is held open by a \\ spring mechanism and can be instantly closed (in order to prevent zooplankton to escape) by pulling or \\ hitting the winch cable. After sampling, the apparatus with open drain cock on which a plankton net has \\ been fixed, is slowly raised over the water surface and thereby emptied and the zooplankton caught. Two \\ men can easily operate this apparatus in spite of its unusually big size.
}

Im Rahmen von Beobachtungen über das Wanderverhalten des Zooplanktons wurde ein Gerät benötigt, das aus definierten Tiefen und möglichst dünnen Wasserschichten genügend grosse, statistisch auswertbare Mengen von Zooplankton lieferte. Um eine Verfälschung des Resultates durch Fluchtreaktionen auszuschliessen, musste die Probenentnahme sehr rasch erfolgen, eine Bedingung, die z. B. für Diaptomiden nicht leicht zu erfüllen ist. Das Probenvolumen musste ferner gegen 1001 betragen; diese Bedingung genügte für die Planktondichte unter sommerlichen Verhältnissen im mesotrophen Vierwaldstättersee.

Aufgrund des Angebotes von handelsüblichen Materialien. insbesondere von Rohrmaterial für den Körper des Wasserschöpfers, wurde ein Modell entworfen, das im Prinzip einem gewohnten Wasserschöpfer zur Entnahme von Seewasserproben für analytische $Z$ wecke ähnelt.

Der mit Profilringen armierte Körper besteht aus einem Polyäthylenrohr mit $415,6 \mathrm{~mm}$ äusserem und $400 \mathrm{~mm}$ innerem Durchmesser; er ist $715 \mathrm{~mm}$ lang (bzw. hoch) und fasst $90 \mathrm{l}$. Die Deckel sind halbiert, die oberen Halbdeckel werden durch ein Gestänge offengehalten und durch seitlich angebrachte Gewichte zur Schliessbewegung gebracht; einmal im Gang, erfolgt diese sodann durch das eigene Gewicht. Die unteren Deckel werden im Bereitschaftszustand durch das gleiche Gestänge offengehalten, welches sie nachher auch schliesst (Abb. 1). Im Gegensatz zu kleineren Wasserschöpfern hängt die gesamte Einrichtung an einem zentralen Stab und ruht im geschlossenen Zustand auf den unteren Halbdeckeln. Dank dieser Konstruktion konnte das Gerät verhältnismässig leicht gehalten werden, ohne an Stabilität und Wasserdichtigkeit einzubüssen. Der Mechanismus geht aus Abb.2 hervor.

Als Auslöser dient eine gefederte Klinke; das gesamte Gewicht hängt an zwei einstellbaren Federn, welche die Klinke blockieren und das Gerät offenhalten (Abb.3). Dieser Mechanismus ist so gestaltet, dass er durch zwei Leute routinemäs- 
sig bedient werden kann, notfalls, geeignete Kraneinrichtung vorausgesetzt, sogar durch einen einzigen Mann. Das Gerät wird nun geöffnet und die Klinke gespannt. In dieser Stellung wird es mechanisch gesichert, bis es zur Probenahme versenkt wird (Abb.4). Mit Vorteil wird das Tragseil des Krans über eine Flaschenzugrolle («Block») doppelt geführt. Das Gerät wird nun ausgeschwenkt, entsichert und abgesenkt. Wird die Absenkbewegung plötzlich gestoppt oder das Tragseil durch einen kräftigen Schlag knapp unterhalb der Kranrolle angeruckt, so wird die Federspannung des Auslösers überschritten, die Klinke, an der das Ganze hängt, freigegeben, und das Gerät schliesst sich unverzüglich (Abb.5).

Es wäre kaum möglich, den vollen Wasserschöpfer über die Wasseroberfläche $\mathrm{zu}$ heben. Da nur das Zooplankton benötigt wird, das Wasser jedoch nicht, ist dies auch gar nicht erforderlich. Der Schöpfer wird deshalb mit geöffnetem Hahn, der ein Zooplanktonnetz trägt, langsam gehoben und das ablaufende Wasser durch das Netz filtriert. Am Schluss wird lediglich der Inhalt des Netzes sorgfältig in den Netztrichter gespült und als Probe gesammelt (Abb.6), worauf der Wasserschöpfer für die nächste Probenahme vorbereitet und wieder versenkt wird.
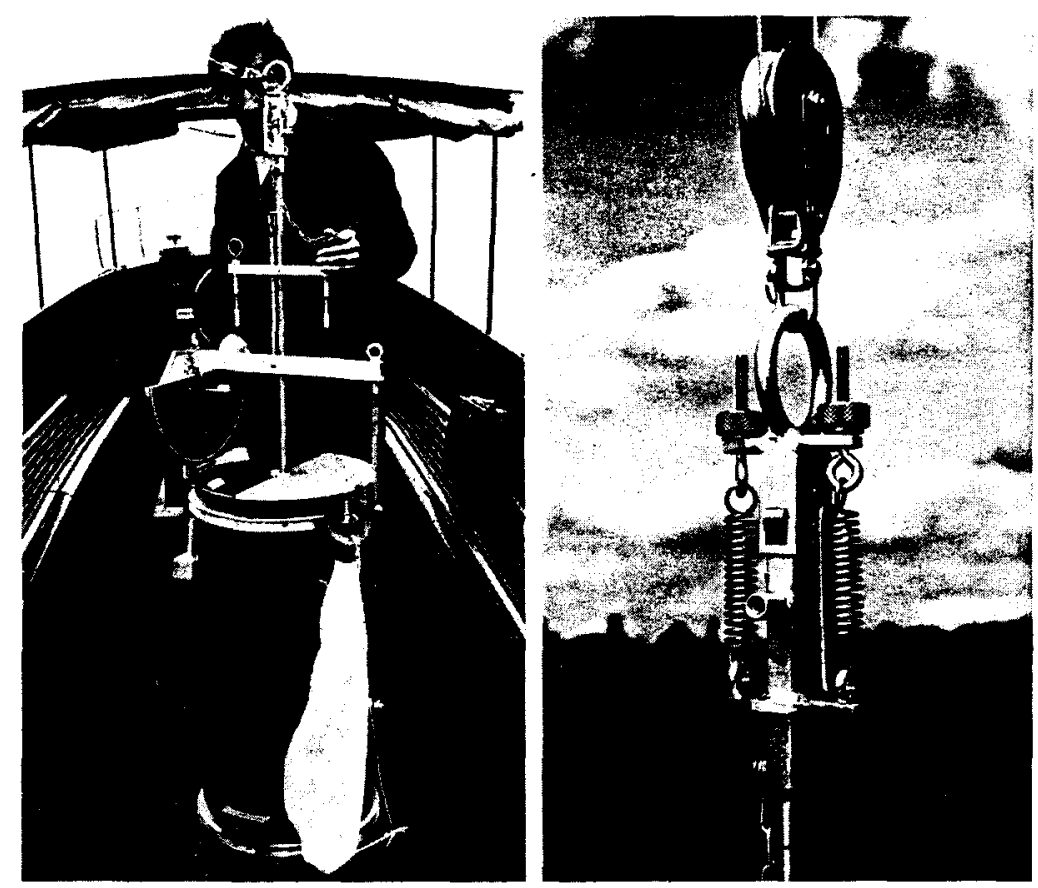

Abb. 1. Wasserschöpfer vor der Probenahme. Das geschlossene Gerät ist knapp mannshoch. Figure 1 . Sampler previous to sampling. The apparatus is approximately $1.5 \mathrm{~m}$ high.

Abb. 3. Auslösemechanismus. Das Gerät hängt jetzt an den Federn. Ein Ruck am Kranseil gibt die Klinke (in Bildmitte) frei. Das geschlossene Gerät hängt sodann am zentralen Tragrohr.

Figure 3. Releasing mechanism. The apparatus is now hanging on the springs. The latch is released by jerking on the winch cable. The closed apparatus hangs on the central shaft. 
Als Materialien wurden Polyäthylen, rostfreier Stahl und Aluminium in handelsüblichen Dimensionen verwendet.

Das Gerät wurde in der Werkstätte der EAWAG (Versuchsstation Tüffenwies) hergestellt. Der Verfasser dankt den Herren H. Schälchli und E. Schwager für die überaus sorgfältige und mitdenkende Konstruktionsarbeit.

H. Ambühl

Adresse des Autors: Prof. H. Ambühl, EAWAG, CH-8600 Dübendorf, Schweiz.
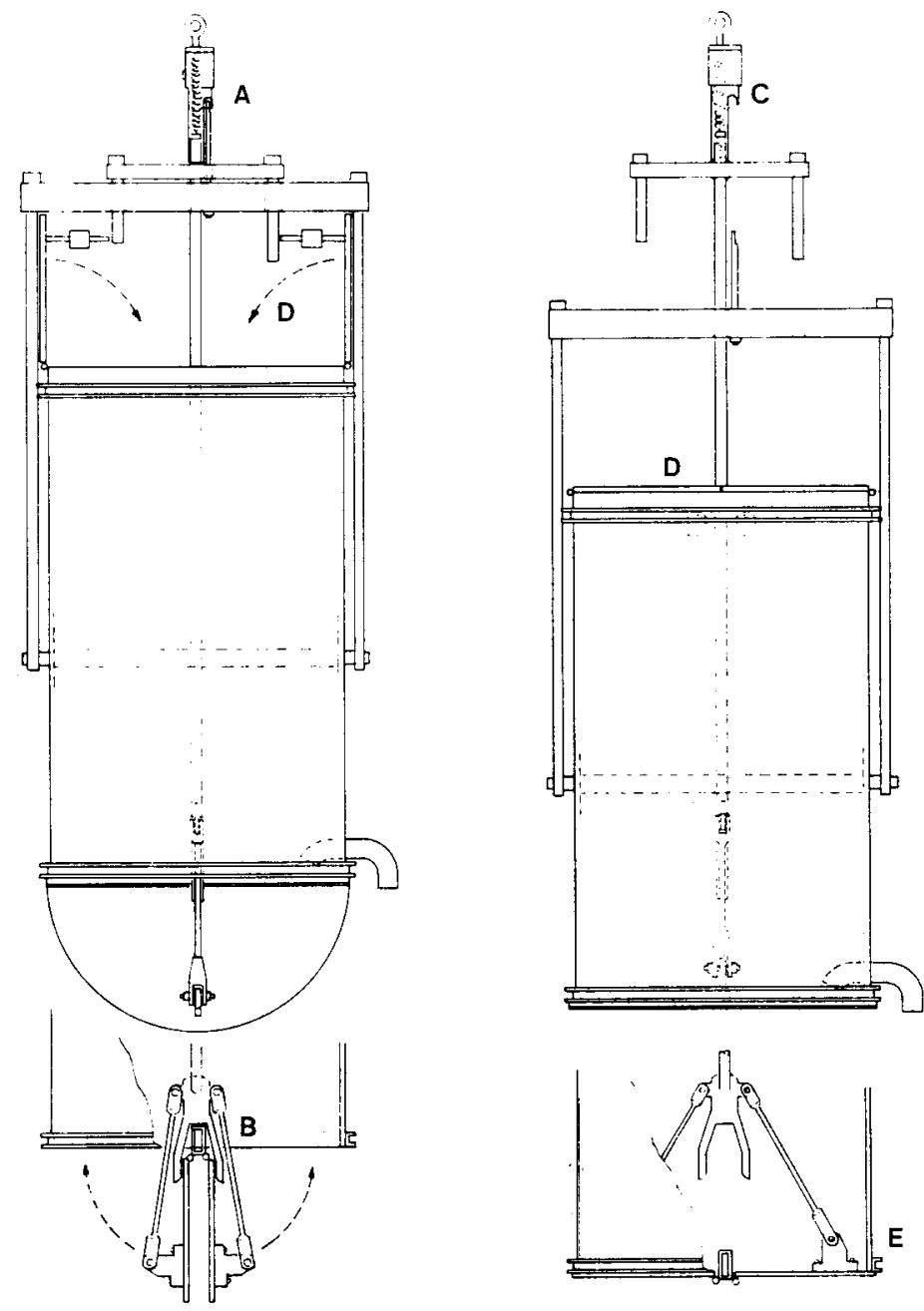

Abb. 2. Konstruktionsskizze. Links: offen; rechts: geschlossen. Der untere Verschlussteil ist separat um $90^{\circ}$ verdreht nochmals dargestellt (Teilskizzen unten).

Figure 2. Schematic drawing. Left: open; right: closed. The lower closing section is drawn again in a $90^{\circ}$ turned view (below). 

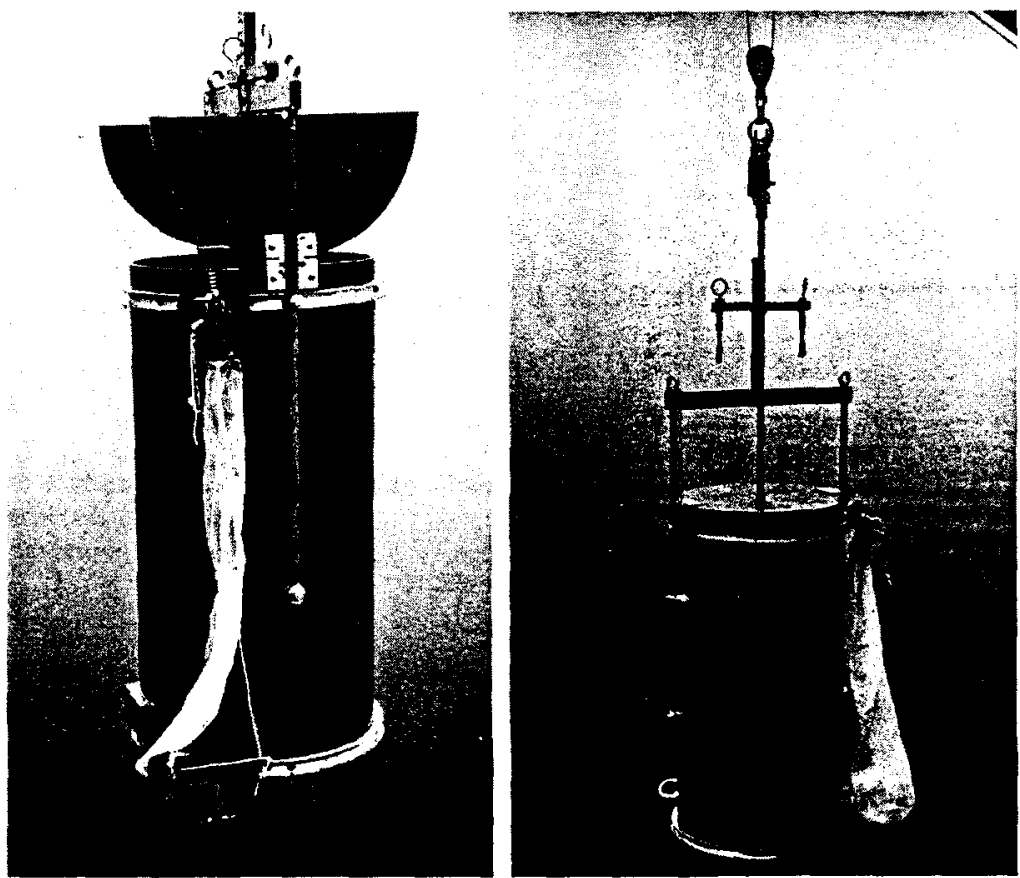

Abb.4. Gerät offen am Kran hängend, vor dem Absenken. Der Korkring dient als Schwimmer, um beim geschlossenen, an die Oberfläche gehievten Gerät den Auslaufstutzen senkrecht zu stellen.

Figure 4. Open apparatus hanging on the winch, previous to lowering. The cork ring serves as a buoy in order to retrieve the line with which the outlet pipe is brought to vertical position.

Abb.5. Gerät geschlossen (aber leer: gefüllt könnte es nicht aus dem Wasser gehoben werden). Figure 5. Apparatus closed (empty; filled it cannot be taken out of the water).

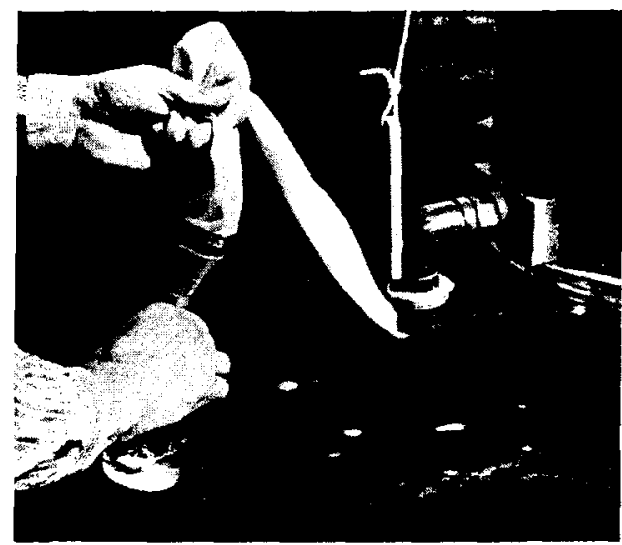

Abb.6. Abfüllen der Planktonprobe aus dem Netz. Figure 6. Sampling plankton from nel. 